\title{
Density-functional calculation of van der Waals forces for free-electron-like surfaces
}

Hult, E.; Hyldgaard, P.; RossmeisI, Jan; Lundqvist, B.I.

Published in:

Physical Review B Condensed Matter

Link to article, DOI:

10.1103/PhysRevB.64.195414

Publication date:

2001

Document Version

Publisher's PDF, also known as Version of record

Link back to DTU Orbit

Citation (APA):

Hult, E., Hyldgaard, P., Rossmeisl, J., \& Lundqvist, B. I. (2001). Density-functional calculation of van der Waals forces for free-electron-like surfaces. Physical Review B Condensed Matter, 64(19), 195414.

https://doi.org/10.1103/PhysRevB.64.195414

\section{General rights}

Copyright and moral rights for the publications made accessible in the public portal are retained by the authors and/or other copyright owners and it is a condition of accessing publications that users recognise and abide by the legal requirements associated with these rights.

- Users may download and print one copy of any publication from the public portal for the purpose of private study or research.

- You may not further distribute the material or use it for any profit-making activity or commercial gain

- You may freely distribute the URL identifying the publication in the public portal 


\title{
Density-functional calculation of van der Waals forces for free-electron-like surfaces
}

\author{
Erika Hult, ${ }^{1}$ Per Hyldgaard, ${ }^{1}$ Jan Rossmeisl, ${ }^{2}$ and Bengt I. Lundqvist ${ }^{1}$ \\ ${ }^{1}$ Department of Applied Physics, Chalmers University of Technology and Göteborg University, S-412 96 Göteborg, Sweden \\ ${ }^{2}$ Center for Atomic-scale Materials Physics (CAMP), Department of Physics, Building 207, Technical University of Denmark, \\ DK-2800 Lyngby, Denmark
}

(Received 13 July 2001; published 26 October 2001)

\begin{abstract}
A recently proposed general density functional for asymptotic van der Waals forces is used to calculate van der Waals coefficients and reference-plane positions for realistic low-indexed Al surfaces. Results are given for a number of atoms and molecules outside the surfaces, as well as for the interaction between the surfaces themselves. The densities and static image-plane positions that are needed as input in the van der Waals functional are calculated self-consistently within density-functional theory using the generalized-gradient approximation, pseudopotentials, and plane waves. This study shows that the van der Waals density functional is applicable to realistic surfaces. The need for physically correct surface models, especially for open surfaces, is also illustrated. Finally the parameters for the anisotropic interaction of $\mathrm{O}_{2}$ with $\mathrm{Al}$ are calculated.
\end{abstract}

DOI: 10.1103/PhysRevB.64.195414

PACS number(s): 68.35.-p, 34.50.Dy

\section{INTRODUCTION}

At large separations the interaction between atoms, molecules, surfaces and other fragments of matter is dominated by the weak but long-ranged van der Waals interaction. This attraction is responsible for a number of phenomena, including adhesion between surfaces and physisorption between an adparticle and a solid surface. It is also very important for less dense matter, such as liquid crystals, polymers, and biomolecular surfaces. The origin of the van der Waals interaction is nonlocal correlation between electrons, which is a nontrivial effect to calculate. Furthermore, the van der Waals energy is usually small compared with the total energy for a typical system. This makes it difficult to treat accurately, and more so the larger the system is. Since it is ever present in all electronic systems, a simple scheme to estimate the van der Waals interaction is very desirable. Density-functional theory ${ }^{1,2}$ (DFT) has proved to be a very useful tool for calculating the ground-state properties of atoms, molecules, and solids. This in principle exact theory requires in practice approximations for the exchange-correlation energy. The localdensity approximation ${ }^{2,3}$ (LDA) is widely used in solid-state physics and different generalized-gradient approximations ${ }^{4-7}$ (GGA's) have in the last years also been used extensively for chemical applications. However, these approximations fail for the long-ranged van der Waals interaction. This is not a failure of DFT itself, but an effect of the local or semilocal nature of the LDA and GGA. To be able to apply DFT to a wider class of materials and phenomena it is essential that the van der Waals interaction be restored into the approximation for the exchange-correlation energy. Recently, a number of studies of van der Waals interactions in DFT have been performed. ${ }^{8-18}$ On the one hand, there have been investigations of how different existing approximations for exchange and correlation mimic the van der Waals interactions, ${ }^{17,18}$ indicating the arbitrariness of the method. In one study, explicit long-range expressions have been added, ${ }^{15}$ showing the need for inclusion of the van der Waals interaction to correctly predict binding energies. In summary, these studies illustrate the need for a general van der Waals density func- tional. On the other hand, a number of van der Waals density functionals have been proposed, within different approaches. One suggests a simple asymptotic functional ${ }^{9}$ that is based on the concept of an effective medium, which leads to a local approximation for the response. It gives surprisingly good results for small objects, but fails for macroscopic bodies. ${ }^{19,10}$ This functional has later been rederived from a different point of view, using a direct local approximation for the response. ${ }^{13}$ The failure for large bodies has then been remedied by introducing more accurate electrodynamics, first only for macroscopic objects, ${ }^{10,20}$ but later for all objects ${ }^{11}$. The resulting unified asymptotic functional, which corresponds to a local approximation to the screened response, has been tested for a large number of different systems, ${ }^{11}$ giving very reasonable results for atoms, molecules, and surfaces. ${ }^{20}$ Another approach uses a local approximation for the Kohn-Sham response of the noninteracting system, which gives a saturated functional when applied to two interacting jellium slabs. ${ }^{16}$ Finally, an approach with calculations in the time domain ${ }^{14}$ is shown to give very accurate results for $\mathrm{He}-\mathrm{He}$ and $\mathrm{He}-\mathrm{H}$ interactions. From all this progress, there is hope that effective tools for the van der Waals interactions in DFT can be developed. To accomplish such a tool, it is important that the limits and applicabilities of the proposed functionals be tested in order to further refine the treatment of long-ranged interactions within a density-functional framework. Of the suggested functionals, the unified asymptotic functional above has been most broadly and extensively tested. ${ }^{11}$ The input needed in this functional is the ground-state electronic densities of the interacting objects and the static polarizabilities for atoms and molecules or image-plane positions for surfaces. These quantities can be calculated within existing density-functional schemes. Earlier tests have used input for real atoms and molecules but only a simple model input for surfaces at different levels of approximations-jellium, stabilized jellium, and $\mathrm{Al}$ surfaces from a quasi-one-dimensional pseudopotential model. ${ }^{21}$ Today's total-energy DFT codes allow quite a realistic description of real surfaces, including possible corrugation, reconstruction, and relaxation. The purpose of this 
study is to show that our proposed general approach to asymptotic interactions is possible to apply in practice to metal surfaces. In this paper the total-energy calculations are performed with the plane-wave pseudopotential DACAPO code. Fully relaxed electron densities are calculated from it, as are the positions of the static image planes $d(0)$. The latter are obtained via self-consistent total-energy calculations in the presence of applied static electric fields of varying magnitudes. The input densities and $d(0)$ values are shown to be in good agreement with other ground-state calculations. For comparative purposes results are also calculated for unrelaxed surfaces. Asymptotic van der Waals interactions between single atoms or molecules and surfaces and between two parallel surfaces are calculated, illustrating the feasibility of the proposed van der Waals densityfunctional scheme. ${ }^{11}$ When it comes to calculated numbers, however, there are unfortunately no results to compare with. We have to content ourselves with the fact that our predictions look very reasonable. The plan of the paper is as follows. In Sec. II the expressions for the van der Waals energy are summarized and the essential approximations for the van der Waals functional given. Calculations of the surface densities and the image planes are described in Sec. III. In Sec. IV the results are given and discussed.

\section{DENSITY FUNCTIONAL FOR ASYMPTOTIC van der WAALS INTERACTIONS}

Mainly for reference purpose, this section gives a summary of available key results for van der Waals interactions at surfaces. Thanks to the work of Zaremba and Kohn ${ }^{22}$ and subsequent studies, ${ }^{23-25}$ the treatment of surfaces is particularly developed. The form of the asymptotic van der Waals interaction between an atom and a surface can be derived from second-order perturbation theory with respect to the atom-surface interaction, assuming negligible overlap of the wave functions of the atom and the surface, giving ${ }^{22}$

$$
E_{\mathrm{vdW}}(z)=-\frac{C_{3}}{\left(z-Z_{0}\right)^{3}} .
$$

The van der Waals coefficient is given by

$$
C_{3}=\frac{1}{4 \pi} \int_{0}^{\infty} d u \alpha(i u) \frac{\epsilon_{B}(i u)-1}{\epsilon_{B}(i u)+1},
$$

and the van der Waals reference plane position is ${ }^{24}$

$$
Z_{0}=\frac{1}{4 \pi C_{3}} \int_{0}^{\infty} d u \alpha(i u) \frac{\epsilon_{B}(i u)-1}{\epsilon_{B}(i u)+1} \frac{\epsilon_{B}(i u)}{\epsilon_{B}(i u)+1} d(i u) .
$$

Here translational invariance along the surface is assumed, and the calculations will be performed for a surface that is averaged over the $x y$ plane. $\alpha(i u)$ is the atomic polarizability for the imaginary frequency $i u$ and $\epsilon_{B}(i u)$ is the bulk dielectric function. Finally,

$$
d(i u)=\frac{\int z \delta n(z, i u) d z}{\int \delta n(z, i u) d z}
$$

is the centroid of the surface charge induced ${ }^{35}$ by a uniform external electric field oriented perpendicular to the surface and varying in time like $e^{u t}$. For jellium, $d(i u)$ has been studied by time-dependent DFT, within the LDA. ${ }^{25,26}$ For two interacting surfaces the interaction must be taken to infinite order, because of the possibility of multiple reflections. The asymptotic form of the van der Waals energy can be written $^{20}$

$$
E_{\mathrm{vdW}}=-\frac{C_{2}}{\left(z-Z_{1}-Z_{2}\right)^{2}},
$$

where the van der Waals coefficient is

$$
C_{2}=\frac{1}{32 \pi^{2}} \int_{0}^{\infty} d u \int_{0}^{\infty} \frac{x^{2} d x}{\frac{\epsilon_{B}^{(1)}(i u)+1}{\epsilon_{B}^{(1)}(i u)-1} \frac{\epsilon_{B}^{(2)}(i u)+1}{\epsilon_{B}^{(2)}(i u)-1} e^{x}-1} .
$$

The van der Waals reference plane positions are given by

$$
\begin{aligned}
Z_{1}= & \frac{1}{32 \pi^{2} C_{2}} \int_{0}^{\infty} d u d_{1}(i u) \\
& \times \int_{0}^{\infty} \frac{x^{2} d x}{\frac{\epsilon_{B}^{(1)}(i u)+1}{\epsilon_{B}^{(1)}(i u)-1} \frac{\epsilon_{B}^{(2)}(i u)+1}{\epsilon_{B}^{(2)}(i u)-1} e^{x}-1},
\end{aligned}
$$

and similarly for $Z_{2}$. The van der Waals coefficients $C_{3}$ and $C_{2}$ depend only on the bulk dielectric functions of the surfaces and are the same for different surface structures, while the van der Waals reference planes depend on the details of the surface. The asymptotic van der Waals functional used in this paper is based on the so-called adiabatic-connection formula $^{3,27,28}$ for the exchange-correlation energy. Two basic approximations are then made: the response is treated at the level of the random phase approximation (RPA), and a local approximation is made for the screened response, which in the RPA is equal to the Kohn-Sham response. In the asymptotic limit this leads ${ }^{10}$ to the correct asymptotic expressions for the van der Waals interaction energy in terms of $\alpha(i u)$ and $d(i u)$. In practice, to calculate $\alpha(i u)$ and $d(i u)$ a local approximation in terms of the electron density is made for the dielectric function of each fragment. This is described in detail in Ref. 11. The polarizability $\alpha(i u)$ must be calculated numerically for each frequency, whereas, because of the simple geometry, for the surface there exists a simple explicit expression for the centroid, ${ }^{10}$

$$
d(i u)=-\frac{1}{\chi(-\infty, i u)} \int z \frac{d \chi(z, i u)}{d z} d z
$$

where

$$
\chi(z, i u)=\frac{1}{4 \pi} \frac{\epsilon(z, i u)-1}{\epsilon_{B}(i u)+1} \frac{2 \epsilon_{B}(i u)}{\epsilon(z, i u)} \Theta(z-d(0)) .
$$

The dielectric function is taken as $\epsilon(\mathbf{r}, \omega)=1-\omega_{p}^{2}(\mathbf{r}) / \omega^{2}$, where $\omega_{p}$ is the local plasma frequency given by the electron 
density, $\omega_{p}^{2}(\mathbf{r})=4 \pi e^{2} n(\mathbf{r}) / m$. As discussed in Ref. 11 the local approximation tends to overestimate the response in the low-density tails. Hence, the response $\chi$ is cut off at $z$ $=d(0)$ to prevent this spurious behavior. ${ }^{10} \mathrm{~A}$ similar condition applies to atoms, where the cutoff is determined from the static polarizability ${ }^{11} \alpha(0)$. The input needed is thus the electron density of the interacting objects and for atoms and molecules the static polarizability $\alpha(0)$ and for surfaces the static centroid $d(0)$, which coincides with the static imageplane position. For the atoms and molecules in this paper, the dynamic polarizabilities necessary for evaluating $C_{3}$ and $Z_{0}$ have been calculated earlier. ${ }^{11}$ In this paper the densities and static image plane positions for the low-indexed surfaces of Al must be calculated, however. This should of course be done for as realistic surfaces as possible. In order to compare with earlier results, values for the unrelaxed surfaces are also calculated.

\section{CALCULATION OF ELECTRON DENSITY AND STATIC IMAGE PLANE}

These calculations of the electron densities and the static image planes are performed within the density-functional theory. The plane-wave pseudopotential code DACAPO, parallelized over both the sampling $k$ points and electronic bands, ${ }^{29}$ is used. The reader is referred to Refs. 30-33 for further details of the DFT method and its plane-wave implementations. Here only some specifications are given. All calculations have been done using the generalized-gradient approximation GGA-PW91 (Ref. 34) for the exchangecorrelation energy. The Kohn-Sham orbitals are expanded in plane-waves and truncated at the cutoff energy 16 Ry. This cutoff energy should be sufficient for the pseudopotentials used, and test runs with 20 Ry show no change in the results. For the $k$-point sampling of the Brillouin zone a MonkhorstPack scheme ${ }^{36}$ is used. To keep down the number of $k$ points used, the true ionic potentials are replaced by pseudopotentials, in this case a norm-conserving GGA pseudopotential for $\mathrm{Al}^{37}$ For the relaxation of the outermost atomic layers a preconditioned quasi-Newton method is used. ${ }^{38}$ In the present work, the $\mathrm{Al}$ surfaces are represented as slabs with 12 Al layers and 12 vacuum layers for the $\mathrm{Al}(100)$ and the $\mathrm{Al}(110)$ surfaces and $12 \mathrm{Al}$ layers and 9 vacuum layers for the $\mathrm{Al}(111)$ surface. The rather large vacuum gap (the smallest corresponds to $17 \AA$ ) is to ensure that the potential step used when creating an applied electric field is placed where there will be no induced electron density. The calculations are performed using 50 irreducible $k$ points in the Brillouin zone for the (111) surface, 36 for the (100) surface, and 35 for the (110) surface. In the relaxation calculations the four central layers of the slab are kept fixed at the bulk positions. The three top layers on each side are allowed to fully relax, whereas the fourth layer is allowed to relax only in the $z$ direction, that is, perpendicular to the surface. From DACAPO the electron density is obtained on a real-space grid. In the asymptotic van der Waals functional only the lateral average $n(z)$ is used. To find the static image plane, needed as input in the van der Waals density functional the centroid $z_{0}$ of the charge $\delta n(z)$ induced by an electric field $E$ applied perpen- dicular to the metal surface is calculated. This centroid

$$
z_{0}=\frac{\int_{-\infty}^{\infty} z \delta n(z) d z}{\int_{-\infty}^{\infty} \delta n(z) d z}
$$

depends on the magnitude of the applied field and determines the location of the image plane when $E \rightarrow 0$. Thus the value for $z_{0}$ when the electric field tends to zero must be calculated. To do this, the applied electric fields should be small enough so that the function $z_{0}(E)$ can be reasonably well fitted by a linear relation $z_{0}=a+b E$. The zero-field imageplane position is then taken as $a$. As an extra check, both positive and negative electric fields are applied (the sign convention is that positive $E$ corresponds to charging the surface positively), and $z_{0}$ is interpolated to zero field. These calculations are done with the atoms fixed in their unrelaxed and relaxed positions. Slabs thinner than 12 atomic layers were first used, but had to be extended to 12 layers to get convergence in the calculations. Finally, the van der Waals coefficients and the reference-plane positions are calculated from Eqs. (2), (3), and (6)-(9). The density $n(z)$ oscillates periodically due to the ion potential. When calculating $C_{3}$ and $C_{2}$ from Eqs. (2) and (6), the average, corresponding to $r_{s}$ $=2.07$ a.u., is taken as the bulk electron density. The integration over $z d \chi(z, i u) / d z$ in Eq. (8) is from far inside the bulk $(z \rightarrow-\infty)$, where the integrand is nonvanishing because of the density oscillations. Hence the function $g\left(z_{c}, i u\right)$ $=\int_{z_{c}}^{\infty} d z z d \chi(z, i u) / d z$ oscillates as a function of $z_{c}$ around a mean value that is attained already for $z_{c}$ just a few atomic units from the surface. This mean value is taken as the value of the full integral. Since $d(i u)$ depends crucially on the surface region, the essence of it should be captured by averaging out the oscillations in the bulk region.

\section{RESULTS AND DISCUSSIONS}

The main point of this paper is to show that our "unified" van der Waals density functional ${ }^{11}$ is feasible for applications to real metal surfaces. Such a study has basically two parts. One is to calculate the input parameters, the electron density and the static image-plane position, and to check that the obtained results are reasonable. The other is to use this input to evaluate the van der Waals density functional in the asymptotic limits. The "input" calculations should be done with full realism. The corresponding total energy should be minimized, which for metal surfaces typically means that they should be relaxed. The effects of relaxation typically are described in terms of a lattice-plane characterization in relation to what the ideal, i.e., unrelaxed, bulk lattice would look like. In Table I the calculated surface-induced changes of the top interplanar distances are compared with other calculations ${ }^{39,40}$ and experimental low-energy electron diffraction data. ${ }^{42-44}$ The small outward relaxations of the $\mathrm{Al}(111)$ and the $\mathrm{Al}(100)$ surfaces found in other studies are reproduced, as are the oscillatory behavior of the $\mathrm{Al}(110)$ multilayer relaxations. The minor discrepancies in the values might be due to the present use of a thicker slab, different number of atoms in each slab layer, and the use of different pseudopotentials. The smallness of the GGA correction for 
TABLE I. Calculated results for the interlayer relaxations $\Delta d_{12}$, $\Delta d_{23}$, and $\Delta d_{34}$, for $\mathrm{Al}$ surfaces, in percent. Results from experiment (marked Expt.) and from other calculations within the LDA and GGA are given for comparison. Bulk interlayer spacings $d_{B}$ are given in column 2 .

\begin{tabular}{lcccc}
\hline \hline & $d_{B}$ (a.u.) & $\Delta d_{12}(\%)$ & $\Delta d_{23}(\%)$ & $\Delta d_{34}(\%)$ \\
\hline$(111)$ & & & & \\
This work & 4.41 & +0.9 & -0.3 & +0.1 \\
Ref. 39 (GGA) & & +1.1 & -0.1 & +0.05 \\
Ref. 40 (LDA) & & +0.8 & +0.5 & \\
Ref. 41 (Expt.) & & $+0.9 \pm 0.5$ & & \\
Ref. 42 (Expt.) & & $+1.7 \pm 0.3$ & $+0.5 \pm 0.7$ & \\
& & & & \\
$(100)$ & & & \\
This work & 3.82 & +1.5 & +0.6 & +0.5 \\
Ref. 39 (GGA) & & +1.7 & +0.6 & -0.4 \\
Ref. 40 (LDA) & & +0.9 & & \\
Ref. 43 (Expt.) & & +1.8 & & -2.4 \\
(110) & & & & -2.8 \\
This work & 2.70 & -8.2 & +4.4 & \\
Ref. 39 (GGA) & & -9.2 & +5.1 & $-1.6 \pm 1.2$ \\
Ref. 40 (LDA) & & -8.5 & +4.8 & \\
Ref. 44 (Expt.) & & $-8.6 \pm 0.8$ & $+5.0 \pm 1.1$ & -1.0 \\
\hline \hline
\end{tabular}

$\Delta d_{12}$ in the (111) surface is likely to be due to its very high electron density. The calculated average electron density distributions $n(z)$ for the relaxed $\mathrm{Al}(111),(100)$, and (110) surfaces are shown in Fig. 1. The oscillatory behavior is apparent. This behavior causes the convergence problems discussed in Sec. III. To be able to compare with other calculations, the values for the static image-plane positions are calculated for both relaxed and unrelaxed surfaces (Table II). However, the calculations for the relaxed surfaces show that the image-plane position "follows' the outermost atomic layer. The values for the image-plane positions, with respect to the outermost layer, are thus the same for the relaxed surfaces, the difference being that the outermost atomic layers have moved somewhat. Figure 2 gives an example of the extrapolation to the zero-field value of the centroid of the induced surface charge for the unrelaxed (110) surface. There have been rather few self-consistent three-dimensional calculations of the screening charge density. Lam and Needs ${ }^{45}$ have calculated the image-plane positions for the (111) and (110) faces, using a pseudopotential technique within the LDA. In that calculation the surfaces are represented by six layers of $\mathrm{Al}$ and six layers of vacuum. Inglesfield ${ }^{46}$ has used an embedded linear augmented planewave (LAPW) method with two layers in the surface region. Compared with these $a b$ initio calculations the image-plane results of the present work are somewhat higher, but the trends over the faces are the same. One-dimensional methods based on jellium do not show the same trends. A calculation using stabilized jellium with face corrugation ${ }^{47}$ gives a value for the image-plane position for the (110) face that is as large as that for the (100) face. For a modified jellium model, which includes laterally averaged pseudopotentials, ${ }^{48}$ the
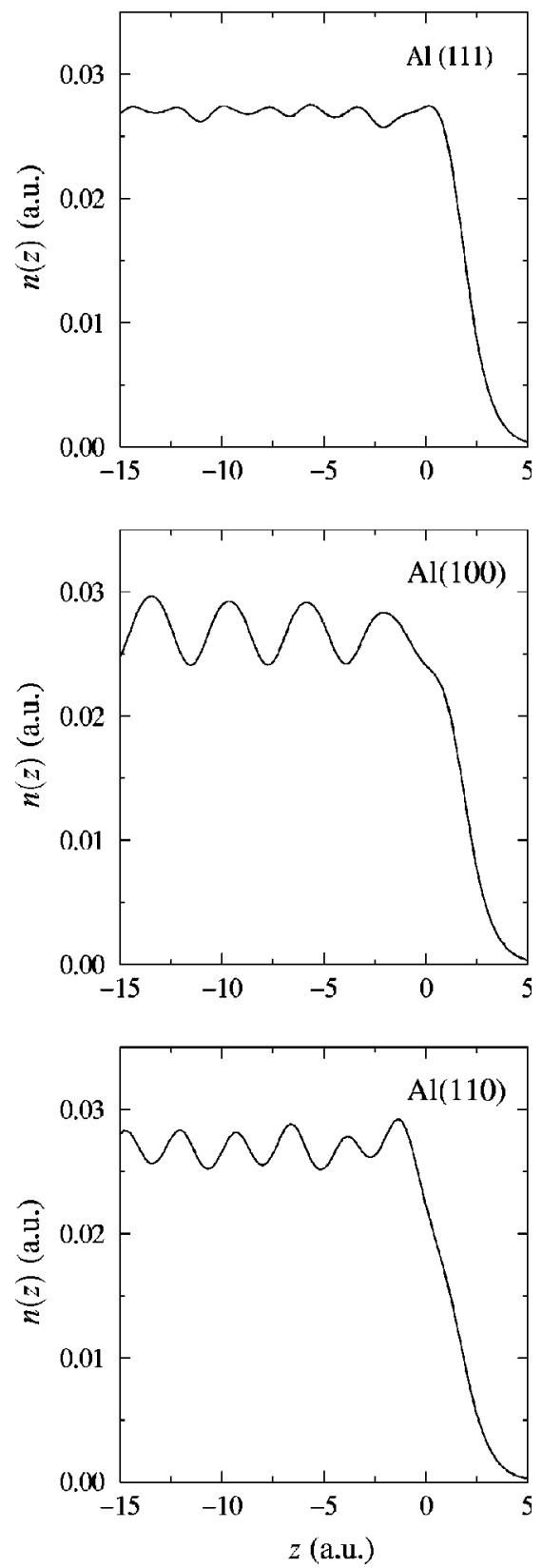

FIG. 1. The calculated average electron density distributions $n(z)$ for the relaxed $\mathrm{Al}(111)$ (top), (100) (middle), and (110) (bottom) surfaces (origin at the outermost layers).

(110) image-plane value is even larger. It should be noted that the one-dimensional calculations do not allow lateral redistribution of the electrons, which may be especially important for the open (110) surface. According to Eq. (1) the van der Waals interaction potential for an atom or a molecule outside a surface has the form $-C_{3} /\left(z-Z_{0}\right)^{3}$. Results for the van der Waals coefficient $C_{3}$ and the van der Waals reference-plane position $Z_{0}$ for a number of atoms and molecules outside the $\mathrm{Al}$ surfaces are displayed in Table III. The origin is taken as the jellium edge for the unrelaxed surfaces; that is, $z=0$ is here taken at half an interplanar spacing outside the outermost atomic layer for the unrelaxed surfaces. The values for $C_{3}$ depend of course on the adparticle and the 
TABLE II. Calculated image-plane positions $d_{\text {im }}$ (a.u.) for the unrelaxed surfaces, taken from the outermost atomic layer. The results are compared with those from other calculations using different treatments of the surfaces.

\begin{tabular}{ccccc}
\hline \hline Face & $d_{\text {im }}$ & ab initio & Modified jellium $^{\mathrm{a}}$ & Stabilized jellium $^{\mathrm{b}}$ \\
\hline$(111)$ & 3.27 & $3.16^{\mathrm{c}}$ & 3.31 & 3.37 \\
$(100)$ & 3.15 & $3.01^{\mathrm{d}}$ & 3.21 & 3.48 \\
$(110)$ & 3.00 & $2.87^{\mathrm{c}}$ & 3.50 & 3.46 \\
\hline \hline
\end{tabular}

${ }^{\mathrm{a}}$ Reference 48 .

${ }^{\mathrm{b}}$ Reference 47.

${ }^{\mathrm{c}}$ Reference 45 .

${ }^{\mathrm{d}}$ Reference 46.

Al bulk properties, whereas the $Z_{0}$ results depend also on the face of the surface. An earlier calculation for atoms and molecules outside stabilized-jellium surfaces ${ }^{49}$ shows a strong face dependence of $Z_{0}$. With the jellium edge as the origin, that calculation gives the largest values of $Z_{0}$ for the open (110) face and the smallest for the close-packed (111) face. The same trend is observed in the present work, however not as pronounced. Especially for the (110) surface the values for $Z_{0}$ are overestimated within the stabilized-jellium model. As is seen from the static image-plane positions, stabilized jellium gives too large a value for the open (110) surface. Since $d(0)$ is used as input into the functional, this of course affects the results. A jellium model with the four outermost

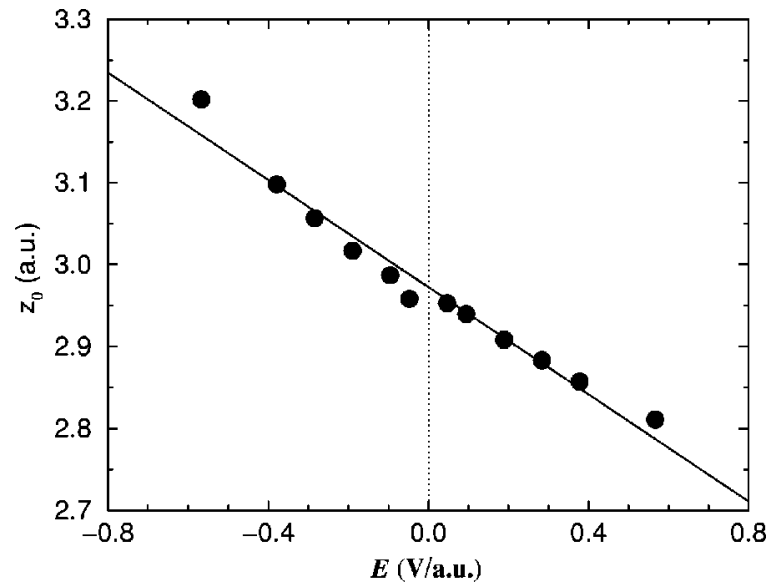

FIG. 2. The position of the center of mass $z_{0}$ of the induced density plotted against the applied field for the $\mathrm{Al}(110)$ surface. The straight line is a linear fit to the calculated values.

layers represented by laterally averaged pseudopotentials ${ }^{21}$ also overestimates the interaction for $\mathrm{H}_{2}$ with $\mathrm{Al}$ surfaces, Table IV. Here, too, the reason is the overestimation of the image-plane position for the (110) surface. In this jellium model, relaxation is taken into account for the (110) surface by placing the two outermost planes in accordance with experimental positions. Relaxation effects do not play a major role, however, as can be seen from Table III. In Table V results for the van der Waals reference-plane positions for

TABLE III. Calculated van der Waals coefficients $C_{3}\left(\right.$ Ry $\left.a_{0}^{3}\right)$ and van der Waals reference planes $Z_{0}$ (a.u.) for atoms and molecules outside the low-indexed $\mathrm{Al}$ surfaces. The origin is taken as the jellium edge for the unrelaxed surfaces, half an interplanar spacing outside the outermost atomic layer of the unrelaxed surfaces.

\begin{tabular}{|c|c|c|c|c|c|c|c|}
\hline & \multirow[t]{3}{*}{$C_{3}$} & \multicolumn{6}{|c|}{$Z_{0}$} \\
\hline & & \multicolumn{2}{|c|}{$\mathrm{Al}(111)$} & \multicolumn{2}{|c|}{$\mathrm{Al}(100)$} & \multicolumn{2}{|c|}{$\operatorname{Al}(110)$} \\
\hline & & Unrelaxed & Relaxed & Unrelaxed & Relaxed & Unrelaxed & Relaxed \\
\hline $\mathrm{He}$ & 0.097 & 0.54 & 0.53 & 0.68 & 0.65 & 0.88 & 0.91 \\
\hline $\mathrm{Ne}$ & 0.21 & 0.49 & 0.48 & 0.63 & 0.60 & 0.81 & 0.84 \\
\hline $\mathrm{Ar}$ & 0.74 & 0.56 & 0.55 & 0.70 & 0.67 & 0.91 & 0.94 \\
\hline $\mathrm{Kr}$ & 1.07 & 0.57 & 0.57 & 0.71 & 0.69 & 0.93 & 0.96 \\
\hline $\mathrm{H}$ & 0.21 & 0.68 & 0.67 & 0.83 & 0.79 & 1.08 & 1.13 \\
\hline $\mathrm{Li}$ & 2.57 & 0.90 & 0.89 & 1.06 & 1.00 & 1.41 & 1.48 \\
\hline $\mathrm{Na}$ & 2.83 & 0.86 & 0.85 & 1.02 & 0.96 & 1.35 & 1.42 \\
\hline K & 4.62 & 0.86 & 0.85 & 1.02 & 0.96 & 1.35 & 1.42 \\
\hline $\mathrm{Be}$ & 1.29 & 0.76 & 0.75 & 0.92 & 0.89 & 1.21 & 1.26 \\
\hline $\mathrm{Mg}$ & 2.13 & 0.78 & 0.77 & 0.93 & 0.88 & 1.23 & 1.29 \\
\hline $\mathrm{Ca}$ & 3.86 & 0.81 & 0.80 & 0.96 & 0.91 & 1.27 & 1.33 \\
\hline $\mathrm{H}_{2}$ & 0.30 & 0.62 & 0.62 & 0.77 & 0.74 & 1.01 & 1.05 \\
\hline $\mathrm{O}_{2}$ & 0.73 & 0.56 & 0.55 & 0.69 & 0.66 & 0.89 & 0.93 \\
\hline $\mathrm{N}_{2}$ & 0.77 & 0.57 & 0.56 & 0.71 & 0.68 & 0.92 & 0.96 \\
\hline $\mathrm{CO}$ & 0.84 & 0.57 & 0.56 & 0.72 & 0.69 & 0.93 & 0.97 \\
\hline $\mathrm{C}_{60}$ & 35.2 & 0.59 & 0.58 & 0.73 & 0.70 & 0.95 & 0.99 \\
\hline
\end{tabular}


TABLE IV. The van der Waals planes $Z_{0}$ (a.u.) for $\mathrm{H}_{2}$ outside the low-indexed Al surfaces (the origin is taken as in Table III). For comparison results $Z_{0}^{J P}$ for jellium with pseudopotentials representing the ions in the three outermost layers, from Ref. 21, are given.

\begin{tabular}{lccc}
\hline \hline Face & \multicolumn{2}{c}{$Z_{0}$} & \multirow{2}{*}{$Z_{1}^{J P}$} \\
\cline { 2 - 3 } & Unrelaxed & Relaxed & \\
\hline$(111)$ & 0.62 & 0.62 & 0.61 \\
$(100)$ & 0.77 & 0.74 & 0.83 \\
$(110)$ & 1.01 & 1.05 & 1.27 \\
\hline \hline
\end{tabular}

interacting surfaces are shown, compared with results from the above-mentioned jellium-based models. Trends with respect to surface dense packing are the same, but the simple models give exaggerations. The differences can be explained by the same reasoning as for $Z_{0}$. Of interest for the dynamics of thermal molecules at metal surfaces is the anisotropic interaction. As a final application of the van der Waals density functional in this paper, the anisotropic interactions between the $\mathrm{O}_{2}$ molecule and the $\mathrm{Al}$ surfaces are given. The van der Waals energy for a homonuclear molecule outside a surface can be expressed as ${ }^{50}$

$$
E_{\mathrm{vdW}}(\theta)=-\frac{1}{\left(d-Z_{0}\right)^{3}}\left[C_{3}^{(0)}+C_{3}^{(2)} P_{2}(\cos \theta)\right],
$$

where $\theta$ is the angle between the molecule axis and the surface normal. The coefficient $C_{3}$ and the van der Waals plane positions are given in Table III. $C_{3}^{(2)}$ is expressed in terms of the anisotropic polarizability, which can be calculated with the van der Waals density functional. ${ }^{11}$ For $\mathrm{O}_{2}$ outside $\mathrm{Al}$ surfaces, this yields $C_{3}^{(2)}=0.072 \mathrm{Ry} a_{0}^{3}$. The rotational anisotropy is thus about $10 \%$, which should affect the dynamics of thermal molecules at metal surfaces. ${ }^{52-56}$ The sticking is at low kinetic energies dominated by the steering effect and sensitive to the rotational quantum number $J$. Such behavior has been observed ${ }^{51}$ and calculated for $\mathrm{H}_{2}$ on $\mathrm{Cu}(110)$ by means of quasiclassical trajectories ${ }^{52}$ and predicted by quantum-dynamical calculations ${ }^{53,55}$ and observed ${ }^{54}$ for $\mathrm{H}_{2}$ on $\operatorname{Pd}(111)$, where typical parameters for the stickingcoefficient measurement are incident translational energy in the range 31-94 meV, rotational states from $J=0-5$, and a surface temperature of $423 \mathrm{~K}$. The sticking coefficient initially decreases with increasing $J$, because more quickly rotating molecules are less easily steered to dissociation channels. ${ }^{52,53}$ It has recently been shown for the growth of $\mathrm{Cu} / \mathrm{Cu}(001)$ that steering may have an important influence on the morphology of growing films. ${ }^{56}$ Steering originates from long-range attractive forces between incoming atoms and substrate atoms and leads to preferential arrival of atoms on top of islands. This general phenomenon should routinely be considered in growth studies. ${ }^{56}$
TABLE V. The van der Waals planes $Z_{1}$ (a.u.) for the interaction between the low-indexed Al surfaces (the origin is taken as in Table III). For comparison results $Z_{1}^{J P}$ for jellium with pseudopotentials representing the ions in the three outermost layers, from Ref. 20, are given, as are results for stabilized-jellium surfaces, $Z_{1}^{S J}$ (input densities and image planes the same as in Ref. 49).

\begin{tabular}{ccccc}
\hline \hline Face & \multicolumn{2}{c}{$Z_{1}$} & \multirow{2}{*}{$Z_{1}^{J P}$} & $Z_{1}^{S J}$ \\
\cline { 2 - 3 } & Unrelaxed & Relaxed & & \\
\hline$(111)$ & 0.72 & 0.71 & 0.67 & 0.80 \\
$(100)$ & 0.89 & 0.85 & 0.84 & 1.06 \\
$(110)$ & 1.16 & 1.21 & 1.29 & 1.40 \\
\hline \hline
\end{tabular}

\section{CONCLUSIONS}

Our van der Waals density functional has previously been tested for simple surface models, where it has proved to work well. Here it is applied to realistic low-indexed Al surfaces, which are self-consistently calculated in three dimensions within DFT. Results in the present work agree with the most realistic from the earlier calculations, as, for example, the result for $\mathrm{H}_{2}$ outside $\mathrm{Al}(111)$. For the less close-packed surfaces, the results illustrate the need for a physically correct treatment. To show trends, simple jellium-based models are useful, but for calculations where a higher accuracy is needed, more realistic surfaces should be used. Similar calculations as in the present work can easily be done for other free-electron-like surfaces. Most of the work is related to calculating the input density and image-plane position. When these quantities exist, the asymptotic van der Waals interaction is easily evaluated. The asymptotic forms (1) and (5) of the van der Waals interaction are singular at small separations and should obviously be corrected for with so-called saturation effects. Such saturation effects seem for many systems to be sizable only at surprisingly small distances. ${ }^{57,58}$ The asymptotic forms might then in some cases be applicable even at separations close to physisorption and adhesion minima, as has been frequently assumed. With shrinking separations the kinetic energy repulsion might dominate the total energy before the saturation condition of the van der Waals attraction has grown to a size of importance. Estimates of the repulsion ${ }^{59}$ indicate that there might be a few such cases.

\section{ACKNOWLEDGMENTS}

Financial support from the Swedish Natural Science Research Council and allocation of computer time at the UNICC facilities at Chalmers University of Technology are gratefully acknowledged. 
${ }^{1}$ P. Hohenberg and W. Kohn, Phys. Rev. 136, B864 (1964).

${ }^{2}$ W. Kohn and L. J. Sham, Phys. Rev. 140, A1133 (1965).

${ }^{3}$ O. Gunnarsson and B. I. Lundqvist, Phys. Rev. B 13, 4274 (1976).

${ }^{4}$ D. C. Langreth and M. J. Mehl, Phys. Rev. B 28, 1809 (1983).

${ }^{5}$ A. D. Becke, Phys. Rev. A 38, 3098 (1988).

${ }^{6}$ J. P. Perdew, in Electronic Structure of Solids '91, edited by P. Ziesche and H. Eschrig (Akademie Verlag, Berlin, 1991).

${ }^{7}$ J. P. Perdew, K. Burke, and M. Ernzerhof, Phys. Rev. Lett. 77, 3865 (1996).

${ }^{8}$ B. I. Lundqvist, Y. Andersson, H. Shao, S. Chan, and D. C. Langreth, Int. J. Quantum Chem. 56, 247 (1995).

${ }^{9}$ Y. Andersson, D. C. Langreth, and B. I. Lundqvist, Phys. Rev. Lett. 76, 102 (1996)

${ }^{10}$ E. Hult, Y. Andersson, B. I. Lundqvist, and D. C. Langreth, Phys. Rev. Lett. 77, 2029 (1996).

${ }^{11}$ E. Hult, H. Rydberg, B. I. Lundqvist, and D. C. Langreth, Phys. Rev. B 59, 4708 (1999).

${ }^{12}$ K. Rapcewicz and N. W. Ashcroft, Phys. Rev. B 44, 4032 (1991).

${ }^{13}$ J. F. Dobson and B. P. Dinte, Phys. Rev. Lett. 76, 1780 (1996).

${ }^{14}$ W. Kohn, Y. Meir, and D. E. Makarov, Phys. Rev. Lett. 80, 4153 (1998)

${ }^{15}$ D. C. Patton and M. R. Pederson, Int. J. Quantum Chem. 69, 619 (1998).

${ }^{16}$ J. F. Dobson and J. Wang, Phys. Rev. Lett. 82, 2123 (1999).

${ }^{17}$ Y. Zhang, W. Pan, and W. Yang, J. Chem. Phys. 107, 7921 (1997).

${ }^{18}$ J. M. Pérez-Jordá, E. San-Fabián, and A. J. Pérez-Jiménez, J. Chem. Phys. 110, 1916 (1999).

${ }^{19}$ Y. Andersson, E. Hult, D. C. Langreth, and B. I. Lundqvist, in Proceedings of the 18th Taniguchi Symposium on Elementary Processes in Excitations and Reactions on Solid Surfaces, edited by A. Okiji, H. Kasai, and K. Makoshi (Springer, Berlin, 1996).

${ }^{20}$ Y. Andersson, E. Hult, P. Apell, D. C. Langreth, and B. I. Lundqvist, Solid State Commun. 106, 235 (1998).

${ }^{21}$ S. Andersson, M. Persson, and J. Harris, Surf. Sci. Lett. 360, L499 (1996).

${ }^{22}$ E. Zaremba and W. Kohn, Phys. Rev. B 13, 2270 (1976).

${ }^{23}$ B. N. J. Persson and P. Apell, Phys. Rev. B 27, 6058 (1983).

${ }^{24}$ B. N. J. Persson and E. Zaremba, Phys. Rev. B 30, 5669 (1984).

${ }^{25}$ A. Liebsch, Phys. Rev. B 33, 7249 (1986).

${ }^{26}$ N. D. Lang and W. Kohn, Phys. Rev. B 1, 4555 (1970).

${ }^{27}$ D. C. Langreth and J. P. Perdew, Solid State Commun. 17, 1425 (1975).

${ }^{28}$ D. C. Langreth and J. P. Perdew, Phys. Rev. B 15, 2884 (1977).

${ }^{29}$ L. Hansen et al., computer code DACAPO-1.30, CAMP, DTU, Denmark.

${ }^{30}$ M. T. Yin and M. L. Cohen, Phys. Rev. B 25, 7403 (1982).

${ }^{31}$ M. C. Payne, M. P. Teter, D. C. Allen, T. A. Arias, and J. D. Joannopoulos, Rev. Mod. Phys. 64, 1045 (1992).
${ }^{32}$ B. Hammer, K. W. Jacobsen, V. Milman, and M. C. Payne, J. Phys.: Condens. Matter 4, 10453 (1992).

${ }^{33}$ G. Kresse and J. Furthmüller, Phys. Rev. B 54, 11169 (1996).

${ }^{34}$ J. P. Perdew, J. A. Chevary, S. H. Vosko, K. A. Jackson, M. R. Pederson, D. J. Singh, and C. Fiolhais, Phys. Rev. B 46, 6671 (1992).

${ }^{35}$ N. D. Lang and W. Kohn, Phys. Rev. B 1, 4555 (1970).

${ }^{36}$ H. J. Monkhorst and J. D. Pack, Phys. Rev. B 13, 5188 (1976).

${ }^{37}$ The GGA Al pseudopotential is constructed by L. Bengtsson, Chalmers University of Technology, using the pseudopotential generator program written by $\mathrm{D}$. Vanderbilt.

${ }^{38}$ Implemented by L. Bengtsson, Chalmers University of Technology. See, e.g., the Boyden-Fletcher-Goldfarb-Shanno algorithm in Numerical Recipes in FORTRAN: The Art of Scientific Computing, 2nd ed., edited by W. H. Press, S. A. Teukolsky, W. T. Vetterling, and B. P. Flannery (Cambridge University Press, Cambridge, England, 1992).

${ }^{39}$ Y. Yourdshahyan, B. Razaznejad, and B. I. Lundqvist (unpublished).

${ }^{40}$ C. J. Fall, N. Binggeli, and A. Baldereschi, Phys. Rev. B 58, R7544 (1998).

${ }^{41}$ H. B. Nielsen and D. L. Adams, J. Phys. C 15, 615 (1982).

${ }^{42}$ J. R. Noonan and H. L. Davis, J. Vac. Sci. Technol. A 8, 2671 (1990).

${ }^{43}$ H. L. Davis, J. B. Hannon, K. B. Ray, and E. W. Plummer, Phys. Rev. Lett. 68, 2632 (1992).

${ }^{44}$ J. N. Andersen, H. B. Nielsen, L. Petersen, and D. L. Adams, J. Phys. C 17, 173 (1984).

${ }^{45}$ S. C. Lam and R. J. Needs, J. Phys.: Condens. Mater. 5, 2102 (1993).

${ }^{46}$ J. E. Inglesfield, Surf. Sci. 188, L701 (1987).

${ }^{47}$ A. Kiejna, Phys. Rev. B 47, 7361 (1993).

${ }^{48}$ P. A. Serena, J. M. Soler, and N. García, Phys. Rev. B 37, 8701 (1988).

${ }^{49}$ E. Hult and A. Kiejna, Surf. Sci. 383, 88 (1997).

${ }^{50}$ J. Harris and P. J. Feibelman, Surf. Sci. 115, L133 (1982).

${ }^{51}$ H. A. Michelsen, C. T. Rettner, and D. J. Auerbach, Phys. Rev. Lett. 69, 2678 (1992).

${ }^{52}$ C. Engdahl and B. I. Lundqvist, Chem. Phys. Lett. 215, 103 (1993).

${ }^{53}$ A. Gross, S. Wilke, and M. Scheffler, Phys. Rev. Lett. 75, 2718 (1995); 77, 405 (1996).

${ }^{54}$ M. Gostein and G. O. Sitz, J. Chem. Phys. 106, 7378 (1997).

${ }^{55}$ A. Gross and M. Scheffler, Phys. Rev. B 57, 2493 (1998).

${ }^{56}$ S. van Dijken, L. C. Jorritsma, and B. Poelsema, Phys. Rev. Lett. 82, 4038 (1999); Phys. Rev. B 61, 14047 (2000).

${ }^{57}$ P. Nordlander and J. Harris, J. Phys. C 17, 1141 (1984).

${ }^{58}$ K. T. Tang and J. P. Toennis, Surf. Sci. Lett. 279, L203 (1992).

${ }^{59}$ See, e.g., F. Montalenti, M. I. Trioni, G. P. Brivio, and S. Crampin, Surf. Sci. 364, L595 (1996). 